better if we are to proactively manage this demand to the benefit of the patient, the ambulance service, and the wider NHS.

1 Schuman LJ, Wolfe H, Sepulveda J. Estimating demand for emergency transportation. Med Care 1977;15:738-49.

2 Sharkey SW, Brunette DD, Ruiz E, et al. An analysis of time delays preceding thrombolysis for acute myocardial infarction. $\mathscr{F} A M A 1989$ 262:3171-4.

3 Birkhead JS. Time delays in provision of thrombolytic treatment in six district hospitals. Join Audit Committee of the British Cardiac Society and Cardiology Committee of the Royal College of Physicians of London. BMF 1992;305:445-8.

4 Rosamond WD, Gorton RA, Hinn AR, et al Rapid response to stroke symptoms: AR, et al. Rapid response to stroke symptoms: the delay in
accessing healthcare (DASH) study. Acad Emerg Med 1998;5:45-51.

5 Lattimer V, George S, Thompson F, et al. Safety and effectiveness of nurse telephone consultaand effectiveness of nurse telephone consulta-

\section{Inappropriate ambulance usage is a retrospective diagnosis}

EDITOR,-In their review of the use of ambulances, Snook et al acknowledge that the vast majority of the existing literature is based on retrospective assessment of medical need and has been undertaken by clinicians in accident and emergency (A\&E) departments after full assessment and diagnosis. ${ }^{1}$ More useful estimates of the level of inappropriate use of emergency ambulances require information about the reason for the emergency 999 call and an assessment of the patient before the definitive diagnosis that is made in A\&E.

It has previously been reported that the most common reasons for inappropriate use of an ambulance are the lack of alternative transports ${ }^{23}$ and alcohol intoxication. ${ }^{4}$ Furthermore, ambulance "misuse" has been reported to be more common among nursing home residents and by the socially deprived. ${ }^{2}{ }^{4}$

Analysis of the cohort of patients not transported to hospital provides additional information about the reasons why patients not requiring care in an $A \& E$ department may call an ambulance. A small audit undertaken by the West Midlands Ambulance Service provides data about patients who were not transported to hospital after a 999 emergency ambulance call. A sample of 100 consecutive report forms, where the patient was not transported, were reviewed. Ambulance patient report forms were analysed from five ambulance stations. These cases were classified reasons could either be clinical or operational.

The reasons for non-removal of a patient from the scene were highly variable. They ranged from automatic fire alarm calls where there may be no incident, to the death of a patient, and from refusal of treatment and transport by the patient to successful treatcontrolled trial. BMf 1998;317:1054-9. according to reason for non-removal. These

ment by the ambulance paramedic (table 1). Those who refused treatment and had minor conditions or no injuries represented $30 \%$ of the study population.

Criteria based dispatch (CBD) has been in full operational use in the West Midlands since April 1997. The CBD categorisation of these patients was reviewed. Of the cases not transported from the scene, $37 \%$ were category A (immediate life threatening situation requiring urgent assistance, requires rapid on scene assistance), $50 \%$ were category $\mathrm{B}$ (serious condition that is not immediately life threatening, requires intervention as soon as possible), and $13 \%$ category $\mathrm{C}$ (non-serious or non-life threatening conditions that require conveyance to hospital). The exclusion of the deceased (need for resuscitation not known until assessment by ambulance crew) still leaves nearly a quarter of cases where maximal resources are deployed and no patient transport was required. More work is needed to determine if CBD could be improved to allow telephone advice rather than dispatch of an ambulance.

SUE WILSON

Emergency Medicine Research Group

University of Birmingham STEVE EDWARDS

West Midlands Ambulance Service

MATTHEW W COOKE

Emergency Medicine Research Group, University of Birmingham and Walsgrave Hospital NHS Trust, Coventry

(Correspondence to: $\mathrm{Dr} M W$ Cooke, Senior Lecturer in Accident and Emergency Medicine, Accident and Emergency Department

Coventry and Warwickshire Hospital, Stoney Stanton Road Coventry CV1 4FH)

1 Snooks H, Wrigley H, George S, et al. Appropriate use of emergency ambulances. $\mathcal{f}$ Accid Emerg Med 1998;15:212-15.

2 Billittier AJ, Moscati R, Janicke D, et al. A multisite survey of factors contributing to medically
unnecessary ambulance transports. Acad Emerg unnecessary ambulance

3 Brady WJJ, Hennes H, Wolf A, et al. Pattern of basic life support ambulance use in an urban pediatric population. Am $\mathcal{F}$ Emerg Med 1996;14 250-3

4 Brown E, Sindelar J. The emergent problem of ambulance misuse. Ann Emerg Med 1993;22: 646-50.

\section{On site computed tomography in the} district general hospital for severe head injury

EDITOR,-In their article in September Dr MacNamara et al clearly indicate the value of 24 hour computed tomography being available to every major accident and emergency (A\&E) department. ${ }^{1}$ They do not, however, indicate in the article whether their scanner is manned fully for the whole of the 24 hours, neither do they indicate the distance and time

Table 1 Patients not transported from the scene
No

Automatic fire alarm, no injured person

Asthma, successfully managed by ambulance crew

Hoax call, no injured person

Psychiatric, successfully managed by ambulance crew (+/- community psychiatric team)

Minor condition or no injury present, successfully managed by ambulance crew

Social problems (for example patient fell out of bed)

Intoxication, no further action required

Violent-removed by police because of danger to ambulance crew

No injury apparent

Diabetic, succesfully managed by ambulance crew

999 call at general practitioner's suggestion, minor condition or no injury

Deceased, resuscitation inappropriate

Refused treatment

Total they are away from their regional neurosurgical unit, although both of these factors are crucial in the development of any local guidelines or protocols involving the method by which these patients should be handled.

Certainly in most district general hospitals it would be hard financially to justify manning of a scanner over the full 24 hour period throughout the year when its likely usage for emergencies out of hours would be quite small. Even allowing for the fact that the figures provided in this article do not allow for weekends and bank holidays, etc, as part of the "out of hours" periods (which must take up a fair number of the 83 annual cases scanned between 0900 and 1700), out of hour usage, even with an A\&E service dealing with 110000 patients a year, was relatively small. Thus I suspect the majority of the district general hospitals operate an "on call" scanning service out of hours and this inevitably will lead to delay in getting the computed tomography unit manned and thus the scan performed.

In certain cases where there is a clear urgent requirement for neurosurgical intervention, this delay-added on to the travelling time between the A\&E department and the neurosurgical centre-could be extremely crucial in determining an outcome.

When a consultant in Wigan (on call computed tomography and between 25 and 40 minutes from the neurosurgical unit) I discussed this matter at length with our local neurosurgeons and we came to the conclusion that in certain specified cases-where there was clear clinical evidence to indicate the need for urgent neurosurgical intervention, such as of a developing intracranial haematoma-it was far preferable to arrange the transfer (with, of course, all normal precautions to prevent secondary brain injury) direct to the neurosurgical unit without local computed tomography. By this means the neurosurgical unit were able to make arrangements to receive the patient during the period of transfer and were thus in a position to assess the patient immediately on arrival, arrange direct transfer to their scanner, and then to theatre if this was indicated. This could save one hour or more in getting such patients to theatre.

These cases aside I would certainly agree that the main benefit of the local scan transmitted to the neurosurgical unit was in those patients that did not fit these criteria. In these cases many unnecessary transfers of seriously ill patients with non-operable conditions were avoided. This often not only involved one transfer but-because of lack of intensive care unit accommodation at the neurosurgical centre-these patients were after computed tomography at the centre, very often either subsequently sent back for care in our own intensive care unit, or transferred to yet another hospital.

I strongly feel that the adoption of any protocol or guideline is a matter of "horses for courses" and, before anyone rushes off to adopt other department's good ideas, the local factors pertaining to such cases should be taken very carefully into consideration since, as in other fields, a course of action which eminently suits one unit could be the potential source of severe hazard to patients in a different environment.

N E SCHOLES

Consultant in Accident and Emergency Medicine, 81 Wigan Road, Standish, Wigan, Lancs WN6 OBQ

1 MacNamara AF, Brazil E, Evans PA. Computed tomography of the head by the accident and emergency department-why 24 hour access is emergency department-why 24 hour acce
vital. $\mathcal{F}$ Accid Emerg Med 1998;15:294-7. 